This item was submitted to Loughborough's Research Repository by the author.

Items in Figshare are protected by copyright, with all rights reserved, unless otherwise indicated.

\title{
Skills in detecting gun carrying from CCTV
}

PLEASE CITE THE PUBLISHED VERSION

PUBLISHER

(C) IEEE

VERSION

VoR (Version of Record)

LICENCE

CC BY-NC-ND 4.0

REPOSITORY RECORD

Blechko, Anastassia, lain T. Darker, and Alastair G. Gale. 2019. "Skills in Detecting Gun Carrying from CCTV". figshare. https://hdl.handle.net/2134/5906. 
This item was submitted to Loughborough's Institutional Repository (https://dspace.lboro.ac.uk/) by the author and is made available under the following Creative Commons Licence conditions.

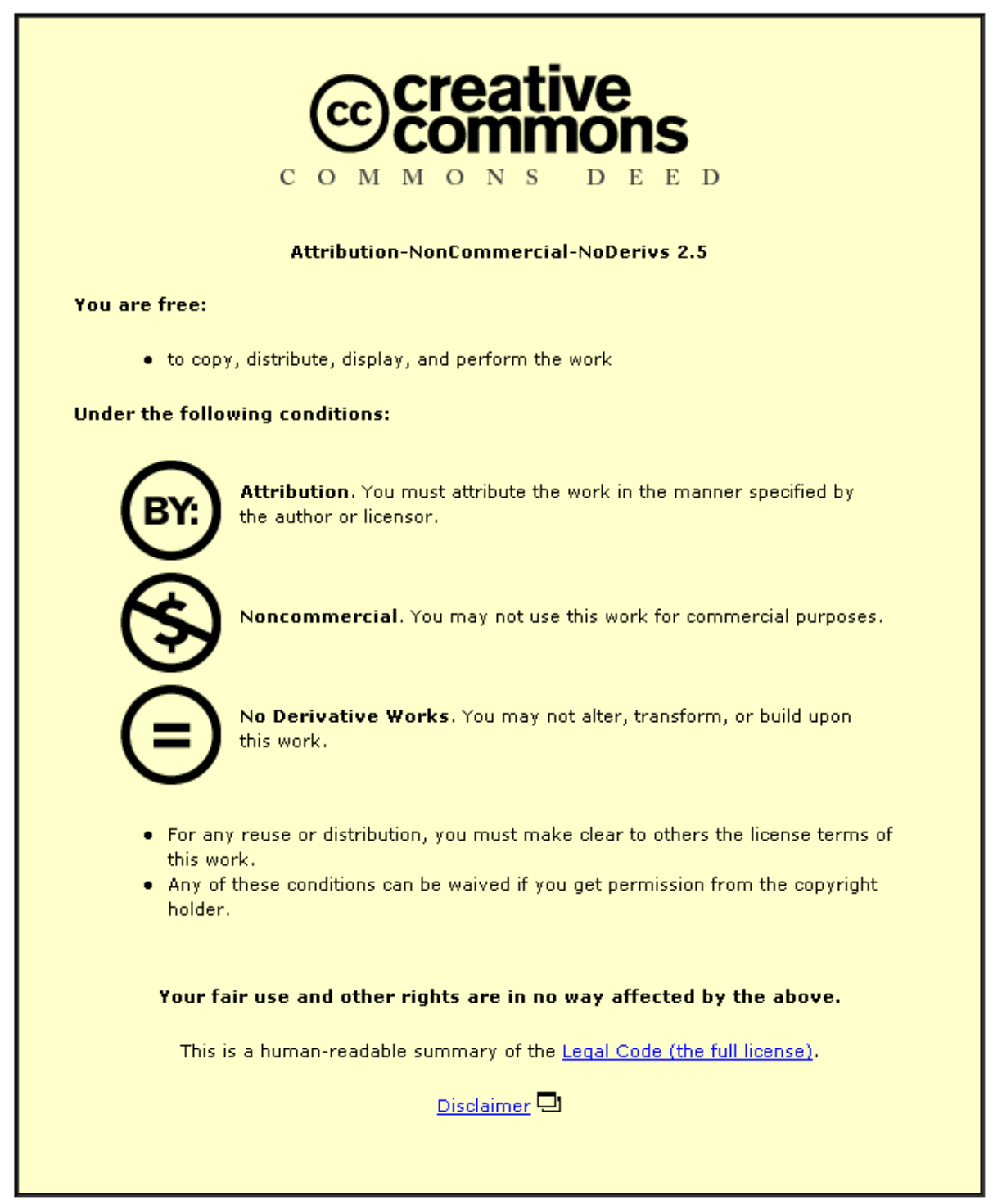

For the full text of this licence, please go to: http://creativecommons.org/licenses/by-nc-nd/2.5/ 


\section{SKILLS IN DETECTING GUN CARRYING FROM CCTV}

\author{
Anastassia Blechko, \\ Applied Vision Research Centre, \\ Centre, \\ University of Loughborough, \\ Loughborough, \\ Loughborough, \\ LE11 3UZ, \\ UK
}

\author{
Alastair Gale, \\ Applied Vision Research \\ University of \\ Loughborough, \\ LE11 3UZ, \\ UK
}

\begin{abstract}
Gun crime is an increasingly common occurrence in the UK. An ongoing research programme is investigating the ability of humans to detect whether or not an individual, captured on CCTV, is carrying a firearm. In the present study we argue that observers respond to cues which individuals inherently produce whilst carrying a concealed firearm. These cues might be reflected in the body language of those carrying firearms and might be apprehended by observers at a conscious or subconscious level. Simulated CCTV footage was generated of individuals who acted as surveillance targets and who carried, concealed on their persons, either firearms or matched innocuous objects. Trained CCTV operators and lay people then viewed this footage and were asked to indicate whether or not they thought the surveillance target was carrying a firearm. The size of the influence of carrying a firearm on a surveillance target's anxiety level was found to be related to the number of times that individual was deemed to be carrying a firearm. However, the surveillance target's anxiety level was not related to sensitivity in firearm detection. Additionally, a test of body language decoding ability did not show that the body language reading skills of observers were related to ability to detect a concealed firearm. These initial results provide some insight into the potential for using a surveillance target's body language to determine if they are concealing a weapon and whether or not such an ability can be acquired through surveillance training and experience.
\end{abstract}

Index Terms - CCTV, Firearm, emotion recognition, nonverbal behaviour

\section{INTRODUCTION}

In the UK, The use of Closed Circuit Television (CCTV) for public space surveillance burgeoned in the 1990s, largely under the auspices of crime reduction [1]. In the course of proactive monitoring, CCTV operators are able to spot malintent, often through the precursors of overtly violent behaviour [2]. Thus, proactive crime detection is one mechanism by which CCTV can act to reduce crime. The late 1990 s and early 2000 s saw a vast increase in the levels of gun crime within the UK [3]. Thus, it is especially desirable detect those who carry firearms on the streets of the UK, via CCTV. However, carrying a firearm is not necessarily accompanied by overtly violent behaviour and the firearm may be concealed to some degree [4]. The present work concentrates on the abilities of CCTV operators to identify potentially subtle behaviours that indicate that someone is carrying a firearm. It has been assumed that the identification of a gun carrier by CCTV operators might be based on body language analysis and particularly on an understanding of emotional state derived from non-verbal behaviour.

Additionally, a potential influence of expertise was investigated by comparing the abilities of CCTV operators with those of lay people. CCTV operators can spend up to 58 per cent of their working time monitoring incidents [5]. As CCTV is a primarily silent medium, it is possible that the ability of CCTV operators to read body language and nonverbal behaviour is highly developed. Indeed, visual information analysis has been found to be one of the core competencies required of CCTV operators $[6,7]$, a component of which is the understanding of body language via CCTV [8].

The act of committing a crime, or preparing oneself to commit a crime, appears to be associated with certain affective processes amongst offenders [9 - 11]. These emotions appear to vary with the type of crime, exhilaration was especially associated with robbery and property crimes, whilst anger was prominent for violent crimes including murder [10]. With respect to firearms offences, seeing a firearm is sufficient to increase the accessibility of aggressive thoughts [12]. This is known as the 'weapons effect' and it is characterised by the ability of a weapon to automatically prime aggression. Thus, it might be inferred that carrying an illegal weapon as a first step towards committing a crime may evoke certain emotions in the would-be offender. Emotional state can be reflected in body language and facial expression $[13,14]$. Consequently, emotions associated with carrying a firearm might result in definitive body movements and facial expressions that can be observed via CCTV.

The present study is part of a larger body of work (the MEDUSA project $[15,16])$ which is investigating strategies used in the detection of concealed and unconcealed firearms for the purposes of deriving an automatic firearms detection system. The present analysis concentrates on the detection of concealed firearms as this task is deemed most likely to involve the use of indirect, behavioural cues.

The experiment reported here focuses upon determining whether visual emotion recognition can support the detection of firearms, via CCTV, and whether this ability is more highly 
developed in CCTV operators. Mock CCTV footage was generated of persons who acted as surveillance targets whilst concealing either a firearm or an innocuous object matched to the firearm. As the weapons effect is automatic, any associated emotions or thoughts evoked by the firearm might be evident in the body language and facial expressions of these surveillance targets. This footage was used as the basis for assessing the abilities of CCTV operators and lay people to detect a firearm, via CCTV, and in relation to the emotional state of the surveillance target. A pilot study using lay people and based on the mock CCTV footage used in the present study found a relationship between the emotional state of the surveillance target, the extent to which that target was deemed to be carrying a firearm, and an observer's sensitivity to the firearm [17].

The abilities of CCTV operators and lay people to detect concealed firearms in video clips derived from this mock CCTV footage were assessed within a signal detection framework. It was hypothesised that CCTV operators would exhibit greater sensitivity in the detection of concealed firearms than lay people. If this hypothesis were accepted it might suggest that the skills involved in this task can be acquired or honed through training or experience.

Evidence of an influence of the emotional state of the surveillance target on the judgements of CCTV operators and lay people regarding the detection of a concealed firearm was also sought. The number of times a surveillance target was deemed to be carrying a firearm and sensitivity to the firearm when carried by that surveillance target were each subjected to correlation with the level of negative affect (in terms of anxiety, depression, and hostility) and positive affect (in terms of positive affect and sensation seeking) experienced across surveillance targets, both whilst the surveillance target was carrying a firearm and whilst the surveillance target was carrying an innocuous object. It is hypothesised that the number of times a surveillance target was deemed to be carrying a firearm would correlate with a metric derived from their emotional state. This would suggest that the emotional state of the surveillance target is manifested in a visually apparent form and that the resultant visual cues are used to inform the decision of the person trying to detect a firearm. These analyses would also allow an assessment of whether reading these emotions might subserve reliable firearm detection.

Finally, sensitivity to the firearm was assessed relative to a standard measure of the abilities of CCTV operators and lay people to read the emotional content of body language and facial expressions (non verbal emotional cues). It was hypothesised that those with greater sensitivity to nonverbal cues would exhibit greater sensitivity to the concealed firearm. If this hypothesis were accepted, it might be inferred that the detection of firearms concealed on the person could proceed on the basis of an interpretation of body language or facial expression.

\section{METHOD}

\section{Participants}

Seventeen people (two groups, CCTV operators and lay people) volunteered to participate in the study relating to the detection of concealed firearms. Data from one participant was discarded as the signal detection model did not account for all the variance in the data. Of the remaining 16 participants, 8 were CCTV operators (age: $M=37$ years, $S D$ $=10$ ) and 8 were lay people (age: $M=47$ years, $S D=12$ ). All the CCTV operators were employed at CCTV control rooms in the UK (years of experience as a CCTV operator: $M$ $=5$ years, $S D=3$ years). The lay people had no training or experience in any sector of the security industry. All participants were naïve to the purpose of the study.

\section{Materials}

Apparatus: Stimulus presentation and data collection were automated using a computer (Toshiba Tecra, Toshiba Ltd.; Microsoft Windows XP version 2002, service pack 2; Genuine Intel $®$ Centrino Duo ${ }^{\mathrm{TM}}$ T2600 processor $2.16 \mathrm{GHz}$; 994 Mhz motherboard; 1.00 GB of RAM) and controlled by a program developed in-house.

The generation of mock CCTV footage and the measurement of sensitivity to a concealed firearm: The mock CCTV footage comprised multiple video clips of 12 actors (surveillance targets), each filmed individually and whilst walking and carrying either a firearm, an innocuous object matched to the firearm for approximate weight and size (a bottle), or no additional object. The present analysis considers only video clips in which the objects (firearms and bottles) were carried concealed on the person.

The levels of negative affect (anxiety, depression, hostility) and positive affect (positive affect and sensation seeking) were assessed for each surveillance target, both whilst carrying a firearm and whilst carrying the innocuous object, using the state version of the Multiple Adjective Affect Checklist - Revised (MAACL-R, [18]). This was achieved within an experiment design that was counterbalanced for order of exposure to the firearm and innocuous object.

The order of clips was randomised with respect to type of object carried (firearm, bottle, none). In the present study two conditions are examined: signal present and signal absent. In the signal present condition, the firearm is featured in the clip, concealed on the surveillance target. In the signal absent condition, the bottle is featured in the clip, concealed on the surveillance target. In terms of signal detection theory, the signal absent condition is equivalent to a noise only condition and it reflects the presence of an object that is not a firearm, whilst the signal present condition is equivalent to a signal plus noise condition and it reflects the presence of an object that is a firearm. An attempt has been made to match the noise in the signal present and signal absent conditions on the basis of the size and weight of the object carried. This is so that the signal reflects visual cues other than those related directly to the presence of a concealed object of a certain size and weight. These cues might be the behavioural consequences of the emotions evoked by carrying a firearm. 
The measure of sensitivity adopted was $d_{a}$, which is a standardised (z-score based) measure of the distance between the means of the noise and noise plus signal frequency distributions, over a range of confidence levels relating to confidence that signal is present or absent. It offers a direct index of sensitivity to the signal, is appropriate when the two distributions are of unequal-variance, and is numerically equal to d' when the two distributions are of equal variance [19].

The Measurement of sensitivity to non-verbal cues: Prior to participating in the firearm detection part of the experiment, each participant undertook a standard test of ability to read body language: the Profile of Nonverbal Sensitivity (PONS) test [20]. Specifically the shortened version of the test was administered and only those portions which address ability to read body language from either the face or body, on the basis of visual cues alone. This version of the test comprised 40 items: 20 face-only and 20 bodyonly items. The items are 10-second video fragments of a 24year-old woman acting in different naturalistic, emotional situations from which the audio content is excluded. After viewing the video fragment the observer is required to make a choice between two descriptions for each video presentation. The observer is instructed to pick the description that best described the situation in which the emotion was portrayed in the previously shown video clip, as quickly and as accurately as possible. Three measures of nonverbal sensitivity were derived from this test: proportion correct across all 40 items; proportion correct across the 20 , face only items; and proportion correct across the 20, body only items.

Design: The experiment was designed to facilitate analyses of sensitivity in concealed firearm detection performance. Of interest were the influences of the expertise of the observer, the ability of the observer to read body language and the emotional state of the surveillance target on sensitivity to the firearm and the decision regarding whether or not a surveillance target was carrying a concealed firearm. Participants were assigned to one of two groups on the basis of their expertise in CCTV surveillance: CCTV operator or lay person.

\section{Procedure}

CCTV operator participants were recruited by contacting CCTV control room managers and through speaking about the study at CCTV practitioner meetings. Lay person participants were recruited by advertising within Loughborough University, from opportunity samples within the locality of Loughborough, and by contacting organisations within Loughborough University who were likely to employ a high proportion of people who could be matched to the previously recruited CCTV operators in terms of age and gender.

The experiment took place over two sessions. Participants were administered the Face and Body PONS test in the first part of the experiment, and the firearm detection test in the second part of experiment.

Session 1 - the PONS test: The aim of the first session was to measure the ability of participants to read body language. Body language reading ability was indexed in terms of ability to decode silent, non-verbal, behavioural cues. The PONS test was automated on a laptop computer. In order to familiarize the participants with the procedure the experiment included a practice session of eight items. The practice session was followed by the 40 items of the main test, given in a randomised order.

Session 2 - the firearm detection test: The firearm detection test was automated on the same laptop computer as the PONS test. Participants were presented with video clips derived from the mock CCTV footage with the aim of measuring their sensitivity to the concealed firearm. Initially, participants viewed eight practice video clips. They then viewed 504 experimental video clips: 72 clips of people carrying concealed firearms; 216 clips of people carrying concealed bottles; 216 clips of people carrying no additional object. The video clips were presented in a pseudorandomised order which spread the signal detection conditions evenly across the time-course of the experiment. After each and every video clip in the firearm detection test, each participant was asked whether or not the person in the clip was carrying a firearm. They indicated their confidence as to whether a firearm was present or absent on a six point scale: "Definitely no"; "Probably no"; "At a guess, no"; "At a guess, yes"; "Probably yes"; and "Definitely yes".

\section{RESULTS}

An a-level of .05 was applied to tests of statistical significance. Statistical analyses were conducted to explore the firearm detection sensitivity of CCTV operators and lay people. Then, potential relationships between the selfreported emotional state scores of surveillance targets and the abilities of observers to recognise when those individuals were carrying a firearm were assessed. Finally, potential relationships between an observer's sensitivity to non-verbal cues and their ability to detect a firearm carrier were assessed.

\section{Expertise and performance on the firearm detection task.}

The frequency of responses across the six response categories in the signal absent and signal present conditions were used to calculate sensitivity to the firearm within a signal detection framework. Performance was indexed in terms of the Receiver Operator Characteristic (ROC) parameter $d_{a}$ which was obtained by means of a maximum likelihood algorithm and based on a Gaussian distribution (RSCORE 5.3.2; [19]).

Sensitivity to the concealed firearm amongst CCTV operators $(M d n=-0.31)$ and lay people $(M d n=-0.44)$ did not differ significantly (Mann-Whitney $U$ test: $U=16.00, p=.105$, $r=-0.42$ ). Consequently, CCTV operators and lay people are considered together for the rest of the analysis. However, it is noted that the trend for CCTV operators have higher sensitivity than lay people in the detection of concealed firearms approached significance (See Fig. 1). 


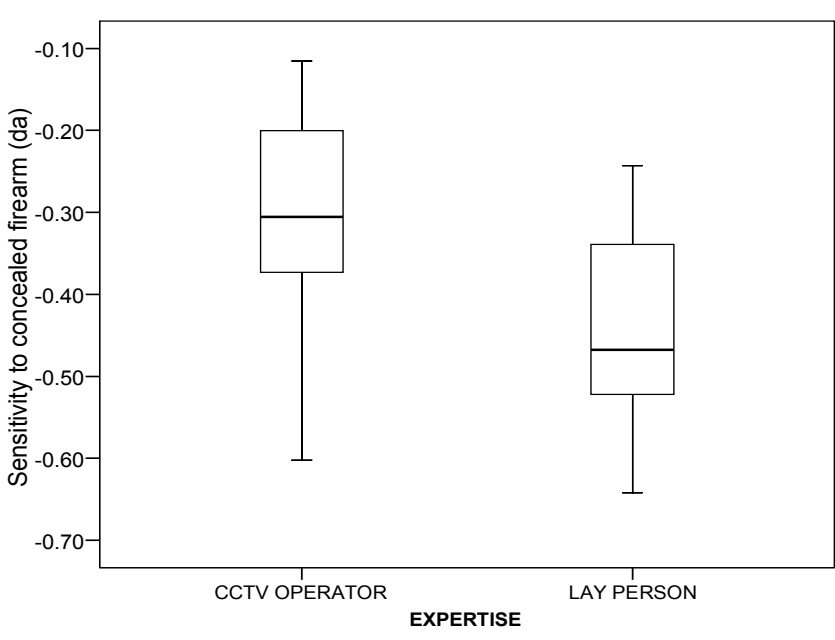

Fig. 1. Sensitivity $\left(d_{a}\right)$ to a concealed firearm amongst CCTV operators and lay people

Correlations between the emotional states of surveillance targets and the frequency with which they were deemed to be carrying a concealed firearm.

Spearman rank order correlations were conducted between the number of times a surveillance target was deemed to be carrying a firearm and the level of affect experienced by that surveillance target, separately for each unique combination of affect scale (anxiety, hostility, depression, positive affect, and sensation seeking) and signal detection condition (signal present and signal absent).

The Spearman rank order correlation tests revealed that there was a significant correlation between the Anxiety scores of surveillance targets in the signal absent condition and the number of times that a given surveillance target was deemed to be a firearm carrier (for data pooled across CCTV operators and Lay people) $\left(r_{s}=0.61, n=12, p=.034\right.$, twotailed). When the same test was performed between the Anxiety scores of surveillance targets and the number of times that a given surveillance target was deemed to be a firearm carrier in the signal present condition (again, for data pooled across CCTV operators and Lay people), no significant correlation was observed, but the result did approach significance $\left(r_{s}=0.5, n=12, p=.098\right.$, two-tailed). No other correlations approached significance.

Spearman rank order correlations were also conducted between the number of times a surveillance target was deemed to be carrying a firearm and the size of the influence of carrying a firearm on the surveillance target's affect level (i.e. a difference between the level of affect experienced by a surveillance target in the signal present and signal absent conditions), separately for each affect scale (anxiety, hostility, depression, positive affect, and sensation seeking).

The size of the influence of carrying a firearm on the surveillance target's anxiety level exhibited a significant, positive correlation with the number of times that that individual was deemed to be a firearm carrier $\left(r_{s}=0.59, \mathrm{n}\right.$ $=12, p=.042$, two-tailed) (see Fig. 2). However, no significant correlations arose on the basis of depression, hostility, positive affect, or sensation seeking.

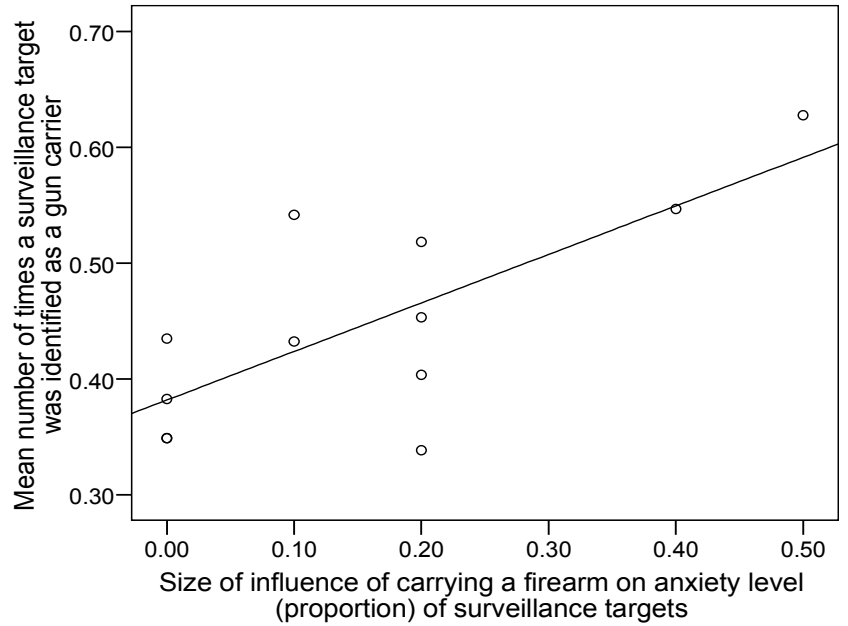

Fig. 2. Correlation between the size of the influence of carrying a firearm on the surveillance target's anxiety level and the number of times that the surveillance target was deemed to be carrying a concealed firearm

Correlations between the emotional states of surveillance target and sensitivity to that target carrying a concealed firearm.

Spearman rank order correlations were conducted between observer's sensitivity to a surveillance target carrying a firearm and the size of the influence of carrying a firearm on the surveillance target's level of affect (i.e., the difference between the level of affect experienced by a surveillance target in the signal present and signal absent conditions), separately for each affect scale (anxiety, hostility, depression, positive affect, and sensation seeking).

The size of the influence of carrying a firearm on the surveillance target's level of anxiety did not influence the sensitivity of an observer to the concealed firearm when carried by that individual. Rather, the size of the influence of carrying a firearm on the surveillance target's sensation seeking level exhibited a significant, negative correlation with the sensitivity of an observer to the concealed firearm when carried by that individual $\left(r_{s}=-0.60, n=12, p=.040\right.$, twotailed). Additionally, no significant correlations arose on the basis of depression, hostility, or positive affect.

\section{Performance on the PONS test}

Performance on the PONS test was indexed as proportion of correct responses, separately in terms of the video clips showing only the face, only the body, and overall, across both types of video clip (see Table I). The results of independent-samples T-tests indicated that there were no significant differences between the scores of CCTV operators and lay people on any of the three summary scores of the PONS test. 
TABLE I

PROPORTION CORRECT ON THE PONS TEST: SUMMARY SCORES FOR CCTV OPERATORS AND LAY PEOPLE

\begin{tabular}{lcccc}
\hline & \multicolumn{2}{c}{$\begin{array}{c}\text { CCTV operators } \\
n=16\end{array}$} & \multicolumn{2}{c}{$\begin{array}{c}\text { Lay people } \\
n=16\end{array}$} \\
\hline PONS score & $M$ & $S D$ & $M$ & $S D$ \\
\hline overall & 0.71 & 0.06 & 0.72 & 0.06 \\
body & 0.72 & 0.11 & 0.69 & 0.11 \\
face & 0.73 & 0.08 & 0.75 & 0.06 \\
\hline
\end{tabular}

Correlations between sensitivity to the concealed firearm and performance on the PONS test

Spearman's correlations were conducted between sensitivity to the concealed firearm and each of the PONS test scores (face only, body only, overall) across participants. Sensitivity to the concealed firearm did not correlate with ability to read body language as assessed using any of the summary scores of the PONS test (body only, $r_{s}=-0.03, n=$ 16, $p=.917$; face only, $r_{s}=-0.26, n=16, p=.340$; Overall, $r_{s}$ $=-0.16, n=16, p=.549)$.

\section{DISCUSSION}

It was found that sensitivity to a concealed firearm did not vary significantly between CCTV operators and lay people. Thus, it appears that training, experience in surveillance, and expertise do not improve performance on the present task. This finding is in accordance with of prior research into the abilities of CCTV operators and lay people to detect malintent via CCTV [2]; it suggests that the task relies upon fundamental human abilities in visual perception and cognition. However, there was a tendency for CCTV operators to exhibit higher levels of sensitivity. The effect was medium-sized and approached significance. Consequently, the experiment may have been underpowered in this respect and a larger sample may have revealed a significant difference; this possibility requires clarification. Even so, sensitivity was below zero for both CCTV operators and lay people. This means that performance was below chance and may indicate that there was a tendency to mistake a concealed innocuous object for a concealed firearm.

The mechanism by which the decision regarding whether or not a surveillance target was carrying a concealed firearm appears to have involved an interpretation of the surveillance target's affective state. In particular, observers' decisions appeared to have been associated with the level of anxiety experienced by the surveillance target. The number of times a surveillance target was deemed to be carrying a concealed firearm was related to their level of anxiety when not carrying a firearm, as well as to the size of the influence of carrying a firearm on their level of anxiety. Additionally, a correlation between the number of times a surveillance target was deemed to be carrying a concealed firearm and their level of anxiety when they were carrying a firearm tended towards significance. Thus, across the five emotional states considered amongst the surveillance targets (anxiety, depression, hostility, positive affect and sensation seeking), anxiety seemed to have the greatest influence the number of times that an individual was deemed to be carrying a concealed firearm. This leads to the conclusion that anxiety was the most influential emotion with respect to observers judgments relating to the carrying of a firearm. Perhaps anxiety is the most visually apparent of the five types of affect considered.

However, it appears that an interpretation of the level of anxiety in a surveillance target does not support reliability in the detection of a concealed firearm on that person. There was no significant relationship between an observer's firearm detection sensitivity and the size of the influence of carrying a firearm on surveillance targets' levels of anxiety. Surprisingly, it was found that the size of the influence of the firearm on the levels of sensation seeking experienced by surveillance targets was correlated negatively with firearm detection sensitivities, across surveillance targets. Thus, it is possible that visual cues related to sensation seeking were used successfully as an indicator of the presence of a concealed firearm; it remains to determine what these visible, behavioural correlates of sensation seeking might be.

These results imply that those engaged in a surveillance task might use visual indicators of the affective state of a surveillance target to inform a decision as to whether or not that individual is carrying a firearm. However, again it is highlighted that the levels of concealed firearm detection sensitivity were consistently below zero, for both CCTV operators and lay people. Therefore, irrespective of any correlations between the affective state of a surveillance target and firearm detection sensitivity, it cannot be inferred that the use of visual indicators of affective state would support reliability in the detection of a concealed firearm.

With respect to the PONS test, it was found that there was no relationship between sensitivity to non-verbal cues and sensitivity to a concealed firearm amongst CCTV operators or lay people. This finding is in accordance with the lack of a strong relationship between the emotional state of a surveillance target and ability to successfully determine whether or not that surveillance target was carrying a concealed firearm. There are a number of potential explanations for this negative finding: the detection of a concealed firearm in the present task does not rely significantly upon ability to read body language; the PONS test does not capture the same aspects of reading body language as those used in the detection of a concealed firearm (the PONS test may not be sensitive [21]); and the detection of a concealed firearm proved too difficult and this factor reduced the opportunity for the reading of body language to contribute to the detection of a concealed firearm. The overall low levels of sensitivity to the concealed firearms would suggest that the latter explanation may be the main factor. 


\section{CONCLUSION}

Prior research has shown that it is possible to use CCTV to detect lawlessness in a surveillance target on the basis of the immediate precursors of overtly violent behaviour [2]. In the present study it was hypothesised that when the surveillance task is to detect a firearm concealed under the clothing of a surveillance target, there may be a reliance on more subtle visual cues (for example non-verbal cues of affective state). The results of the present study give support to this hypothesis. Higher level of anxiety in a surveillance target seems to be the most prominent cues used by observers in the decision process about the presence of a concealed firearm. However, anxiety levels did not appear to support reliable concealed firearms detection. Rather, sensation seeking in the surveillance target allowed a better differentiation between those who were carrying concealed firearms and those who were not. Even so, performance in the detection of a concealed firearm was consistently below chance. Consequently, affective state was not used to reliably infer the presence of a concealed firearm.

However, the shortcomings of the present study should be considered before dismissing the potential importance of visible indicators of affect in the detection of concealed firearms. The present study relied upon the use of mock CCTV footage with actors as surveillance targets. Real-life situations involving those with a genuine intent to use or conceal a firearm might provide a great wealth of visual cues to the presence of a firearm [15, 16].

The present study raises a number of questions to be addressed by future research. It has been demonstrated that the emotional state experienced by an individual whilst carrying a concealed firearm can be linked to the detection of a concealed firearm on that person, when they are observed via CCTV. It remains to be determined how these visual cues influence the decision making process. For instance, they may operate overtly at a conscious level, or they may operate covertly at a subconscious level [16]. The level at which these cues operate might have implications with regard to how to train surveillance professionals to spot concealed firearms (or other items of interest); indeed if such cues prove misleading whilst operating at a subconscious level, it might prove difficult to get surveillance operatives to disregard such sources of information. However, the present study may have had limited sensitivity with respect to behavioural cues to the presence of a concealed firearm (by virtue of the use of mock CCTV footage). Thus, it also remains to be determined whether, and if so under what circumstances, the perceived affective state of a surveillance target can support the reliable detection of a concealed firearm. The use of real-life footage CCTV footage might have supported higher sensitivity in the detection of concealed firearms. Further, the use of real-life CCTV footage, perhaps alongside a larger sample size, might have revealed a significant advantage for CCTV operators in the surveillance task, rather than just a trend which approached significance in that direction.

In the present study it has been found that the decision regarding whether or not a surveillance target is carrying a concealed firearm is related to the emotional state of that surveillance target. It is inferred that this phenomenon relates to the use, either at a conscious or subconscious level, of visual indicators of another's emotional state. Future research should address whether this phenomenon could be honed to support the reliable detection of subtle cues that might indicate that a surveillance target is carrying a concealed firearm.

\section{ACKNOWLEDGEMENTS}

The MEDUSA project is funded by the EPSRC (grant number: EP/D078105/1).

\section{REFERENCES}

[1] C. Norris and G. Armstrong, The Maximum Surveillance Society: The Rise of CCTV. London, Berg, 1999.

[2] T. Troscianko, A. Holmes and J. Stillman, "What Happens Next? The Predictability of Natural Behaviour Viewed Through CCTV Cameras." Perception, vol. 33, pp. 87-101, 2004.

[3] D. Povey (Ed.), K. Coleman, P. Kaiza, J. Hoare, and K. Jansson. Homicides, Firearm Offences and Intimate Violence 2006/07. (Supplementary Volume 2 to Crime in England and Wales 2006/07). Home Office Statistical Bulletin 03/2008.

[4] G. Hales, C. Lewis and D. Silverstone, "Gun Crime: The Market In and Use of Illegal Firearms," Home Office Research Study 298, London, Home Office, 2006.

[5] M. Gill, A. Spriggs, J. Allen, M. Hemming, P. Jessiman, D. Kara, J. Kilworth, R. Little, D. Swain. Control room operation: findings from control room observations. Home Office Online Report 14/05, 2005.

[6] E. Wallace, and C. Diffley. CCTV: Making it work. Recruitment and selection of CCTV operators. Publication No. 8/98. Home office police scientific development branch. United Kingdom, 1998.

[7] C.H.M. Donald. Vigilance. In: People in Control: Human Factors in control room design. Eds. J. Noyes, and M. Bransby, pp. 35-50, 2001.

[8] C.H.M. Donald. Learn to see the body talk. CCTV image. CCTV User Group, p. 28, 2006

[9] M. Cusson. Situational deterrence: fear during the criminal event. Crime prevention studies, 1, pp. 55-68, 1993.

[10] D.V. Canter and M. Ioannou. Criminals' emotional experiences during crimes. International Journal of Forensic Psychology, 1 (2), pp. 71-81, 2004.

[11] J. Katz. Seduction of Crime: Moral and Sensual Attractions in doing Evil. USA: Basic Books, 1988.

[12] C.A. Anderson, A. J. Jr. Benjamin \& B.D. Bartholow. Does the gun pull the trigger? Automatic priming effects of weapon pictures and weapon names. Psychological Science, 9(4), pp. 308-314, 1994.

[13] P. Ekman, and W.V. Friesen. Head and body cues in the judgment of emotion: a reformulation. Perceptual and Motor Skills, 24, pp. 711-724, 1967.

[14] F.E. Pollick, H.M. Paterson, A. Bruderlin, and A.J. Sanford. Perceiving affect from arm movement. Cognition, 82, B51-B61, 2001.

[15] I.T. Darker, A. Gale, L. Ward \& A. Blechko. Can CCTV reliably detect gun crime? In S. Sanson (Ed.), 
Proceedings of the 41st IEEE International Carnahan Conference on Security Technology (pp. 264-271). Piscataway, NJ; IEEE, 2007.

[16] I.T. Darker, A. Gale, L. Ward, A. Blechko \& K. Purdy. Light, Camera, Action, and Arrest. Contemporary Ergonomics 2007. P.D. Bust (Ed.). Taylor and Francis, London, pp. 171-177, 2007.

[17] A. Blechko, I.T. Darker, and A.G. Gale. Exploring essential skills of CCTV operators: the role of sensitivity to non-verbal cues. Contemporary Ergonomics 2008. P.D. Bust (Ed.). Taylor and Francis, London, pp. 145150, 2008.

[18] B. Lubin, and M. Zuckerman. Manual for the MAACL-R: Multiple affect adjective check list - revised. Educational and Industrial Testing Service, San Diego, California, 1999.

[19] L.O. Jr. Harvey. 'RSCORE 5.3.2". Department of Psychology, University of Colorado, Boulder, Colorado, 2001.

[20] R. Rosenthal, J. A. Hall, M. R. DiMatteo, P. L. Rogers, \& D. Archer. Sensitivity to nonverbal communication: The PONS test. Baltimore: Johns Hopkins University Press, 1979.

[21] N. Meiran, T. Netzer, S. Netzer, D. Itzhak, and O. Rechnitz. Do tests of nonverbal decoding ability measure sensitivity to nonverbal cues? Journal of Nonverbal Behaviour, 18 (3), pp. 223 - 244, 1994.

\section{VITA}

Anastassia Blechko graduated from the University of Amsterdam, the Netherlands, in 2001 with an MA degree in cognitive psychology. She is a member of the European Chapter of the Human Factors and Ergonomics Society, the Ergonomics Society (the UK), the Dutch Association for Psychonomy (NVP), and the Applied Vision Association. Since 2006 Anastassia has been a Research Student allied to the MEDUSA project.

lain Darker graduated from Nottingham University, England, with a BSc in neuroscience and a PhD in cognitive psychology. He is a member of the Institute of Engineering and Technology and the Applied Vision Association. Since 2006 lain has been a Research Associate on the MEDUSA project.

Alastair Gale graduated from the University of Durham, England, with a BSc and a $\mathrm{PhD}$ in psychology. He is a Fellow of the British Psychological Society, a Chartered Psychologist and Health Psychologist, a Registered Ergonomist, and a Fellow of the Ergonomics Society. He is also an honorary member of the Royal College of Radiologists' Breast Group and was awarded honorary membership of the Royal College of Radiologists in 1998 for his radiological research. Alastair has been Professor of Applied Vision Sciences at Loughborough University, England, since 2005. 